\title{
Juventud e investigaciones sociales en Cuba
}

\author{
María Isabel Domínguez*
}

\begin{abstract}
Resumen
Los estudios sobre Juventud han constituido una de las áreas de tratamiento más sistemático y estructurado por parte de las Ciencias Sociales en Cuba. El siguiente artículo hará referencia al recorrido de dichos estudios por más de cuatro décadas y centrará la atención en el tratamiento de un problema concreto investigado en la última etapa: la integración social de la juventud en el país y el impacto de los resultados de esos estudios en los cambios en las políticas sociales dirigidas a este sector.
\end{abstract}

Palabras clave: Juventud, Investigaciones Sociales, Integración Social, Política Social, Subjetividad, Identidad Generacional.

\begin{abstract}
The studies about Youth have constituted one of the more systematic and structured treatment area for Social Sciences in Cuba. The present paper makes reference to the journey of these studies for more than four decades. Also it pays attention to the treatment of a concrete problem researched in the last stage: the youth's social integration in the country and the impact of the results of those studies in the social policies to this sector and its transformations.
\end{abstract}

Key words: Youth, Social Research, Social Integration, Social Policy, Subjectivity, Generational Identity.

* María Isabel Domínguez García es Licenciada en Sociología, Doctora en Ciencias Sociológicas, Profesora Titular, Investigadora Titular. Especialidad de trabajo: Sociología. Entidad: Centro de Investigaciones Psicológicas y Sociológicas (CIPS). Cargo: Jefa del Grupo de Estudios sobre la Juventud. Académica Titular de la Academia de Ciencias de Cuba. midominguez@ceniai.inf.cu 


\section{Los estudios sobre juventud en Cuba}

Los estudios sobre Juventud han constituido una de las áreas de tratamiento más sistemático y estructurado por parte de las Ciencias Sociales en Cuba en las últimas cuatro décadas en correspondencia con elementos tales como el alto peso que ha tenido este grupo social en la estructura demográfica de la población que, en períodos como la década de los años ‘ 80 , constituyó más de la tercera parte del total y, unido a la niñez, representaron más del $50 \%$ de la población, así como su significativo papel en los procesos sociales más importantes que han tenido lugar en la historia del país y la prioridad que le ha brindado la política social en áreas esenciales como salud, educación y empleo.

Sin embargo, antes de 1959 (triunfo de la Revolución), no puede hablarse de investigaciones sobre la juventud, entendiéndolas como estudios de distintas disciplinas científicas, tal como ocurrió con la mayor parte de los temas sociales. El lapso 1959 y 1968 fue un período de formación de profesionales y organización de las bases para dar inicio paulatino a la investigación social en el país; pero, en el tema de la juventud, hubo un tratamiento amplio en el pensamiento político que señaló importantes bases para formulaciones teóricas posteriores.

Según una periodización hecha por la autora (Domínguez, 1995) -y actualizada para esta ocasión- es posible identificar tres etapas en el desarrollo de las investigaciones sociales sobre juventud:

1. 1969-1985: Surgieron los dispositivos institucionales para abordar la temática y tuvieron lugar los primeros estudios. Su rasgo fundamental fue la dispersión, concentrados muchas veces en temas muy específicos o en universos demasiado pequeños y localizados, con fuerte predominio del empirismo y muy pocos esfuerzos por desarrollar una concepción teórica. Hubo una cierta identificación entre juventud y estudiantes, los que constituyeron el centro de casi todos los análisis efectuados. 
En los años finales de esa etapa, es decir, entre 1981 y 1985, se dieron los primeros pasos para eliminar la dispersión existente, a partir de la constitución de "Problemas de Investigación" que reagrupaban los esfuerzos en torno a aspectos importantes de la problemática juvenil. Muchas de estas investigaciones trabajaron con muestras nacionales y trataron de brindar una imagen menos local y circunscrita, aunque mantuvieron el énfasis en el grupo de los estudiantes.

En esa etapa, predominaron los enfoques psicológicos y psicopedagógicos en la investigación y, en esas áreas, se obtuvieron algunos avances, pero otras disciplinas apenas estuvieron presentes.

El rasgo principal del período fue el de realizar un elevado número de estudios empíricos centrados en la conducta y en rasgos de la conciencia de los jóvenes, generalmente orientados hacia el grupo de estudiantes, pero con poca atención sobre la acción de las instituciones socializadoras, con un patrón metodológico basado en las encuestas, que condujeron poco a la reflexión teórica y a las interpretaciones globales. Su valor fundamental fue el papel que jugó en la acumulación de información y en la preparación de profesionales.

2. 1986-1994: Esta etapa se caracterizó por la organización de Programas Nacionales de Investigación sobre la Formación de la Juventud, con el propósito de coordinar esfuerzos para integrar los distintos estudios en un proyecto más amplio, a la vez que contribuyó a una priorización de la temática dentro de las investigaciones sociales.

El primer programa se desarrolló entre 1986 y 1990 y su principal objetivo estuvo dirigido a completar un diagnóstico de la juventud lo más amplio posible y en dos direcciones fundamentales:

- Una caracterización de su estructura social en términos socioclasistas y generacionales que profundizara en cada uno de los grupos que la conformaban, tanto en sus características estructurales como en rasgos de su subjetividad. 
- Una caracterización del proceso de socialización que estudiara la influencia de cada una de las instituciones socializadoras fundamentales.

El segundo programa (1991-1994) se planteó, como objetivos, superar la tendencia al carácter descriptivo de las investigaciones, con un mayor énfasis en las funciones interpretativas y de pronóstico. Igualmente, se recomendó incrementar la utilización de métodos y técnicas cualitativas en la recopilación y análisis de la información, diseñar y desarrollar métodos y técnicas de cambio y desarrollar la reflexión teórica sistemática sobre el papel de la juventud y la formación de valores en la sociedad cubana contemporánea. Estos objetivos generales se irían concretando en líneas que dieran respuesta a las demandas más inmediatas que planteaba la situación económica y social del país y que ejercían su efecto sobre la juventud ${ }^{1}$.

Esta segunda etapa significó un acopio de conocimientos importantes en el plano teórico y metodológico; en el de la evaluación de la experiencia internacional; en el de la situación concreta de la juventud cubana y sus instituciones socializadoras y en el de las vías para la introducción de los resultados a la práctica social. Se abarcó el universo juvenil en su conjunto, tomando en cuenta su diversidad interna y se estudió en comparación con otras generaciones.

3. 1995 - a la actualidad: Esta última etapa se ha caracterizado por dos tendencias fundamentales:

- Conservación de la prioridad del tema dentro de las líneas estratégicas para el desarrollo de las Ciencias Sociales en el país².

1 Recordar que la primera mitad de los años ' 90 fue la etapa más crítica de la crisis económica que sufrió el país como resultado de la pérdida de sus vínculos con el campo socialista de Europa Oriental después de la caída del Muro de Berlín y por el recrudecimiento del bloqueo de Estados Unidos. Fue la etapa conocida en Cuba como "Período Especial".

2 Por ejemplo, está indicada entre las prioridades que identifica la Resolución 132 del Ministerio de Ciencias, Tecnología y Medio Ambiente que norma las líneas estratégicas para las Ciencias Sociales, a la vez que se encuentra entre las líneas principales a las que ha convocado el Programa Nacional Científico - Técnico (PNCT) "Sociedad Cubana". 
- A pesar de esa prioridad, se aprecia cierto retorno a la dispersión de los estudios, pues el hecho de no existir un mecanismo que los nuclee, como fue el caso de los Programas, hace que los esfuerzos de las distintas instituciones no logren integrarse plenamente, aun cuando la madurez y estabilidad en el tiempo de dichas instituciones y grupos de trabajo favorecen la continuidad de la investigación y su utilización.

En el momento actual, se dan pasos entre distintas instituciones de Ciencias Sociales del país para articular esos esfuerzos dispersos a través de la creación de una red nacional sobre juventud y la elaboración de una base de datos que permita conocer qué se está haciendo sobre el tema, así como repensar las líneas de interés en los estudios sobre este grupo social y su colocación en la agenda de prioridades nacionales, territoriales y de instituciones de investigación. Ello podría dar lugar a una nueva etapa en la evolución de las investigaciones sobre juventud en Cuba.

A continuación, haré referencia a una de las principales investigaciones sobre juventud realizadas en la última etapa y referida al tema de su integración social (Domínguez, Cristóbal y Domínguez, 2000, 2002, 2004, 2005).

\section{La integración social de la juventud cubana a partir de los años '90. Un estudio}

El escenario de los años '90 para la sociedad cubana se caracterizó por la profunda crisis económica que afectó al país y por importantes cambios resultantes de la estrategia de enfrentamiento seguida para salir de ella, basada en la difícil combinación de elevar la eficiencia económica con la menor afectación de los niveles de justicia social alcanzados.

La convergencia de todo este conjunto de factores produjo diversos efectos sociales que han tenido una influencia más directa o más mediatizada sobre la juventud, sin olvidar que ello 
está condicionado, en gran medida, por las condiciones de partida; en particular el nivel de integración social previo y el grado de preparación que tenía la población para enfrentar una situación de crisis.

Es necesario apuntar que, cuando hablamos de la juventud en Cuba, nos estamos refiriendo al segmento de población entre 14 y 30 años, un sector que -a pesar de la considerable reducción de sus efectivos en más de 300 mil personas en la década de los ‘90 como resultado de la transición demográfica que tiene lugar en el país, lo que representó un decrecimiento del 10\% del grupo juvenil- aún representa el 24,8\% del total de la población (ONE, 2005: 211).

En estas condiciones de partida, confluyeron elementos favorables y desfavorables. El más positivo ha sido, sin duda, el fuerte consenso en torno a valores básicos como la igualdad y la justicia, que ha mantenido a la mayoría integrada al proyecto social y la capacidad creativa y de resistencia que forma parte de la identidad del cubano ${ }^{3}$.

Entre los principales elementos negativos, habría que mencionar la disminución de la participación y el desarrollo de una conciencia igualitarista, que provocó un disparo de las expectativas de los diferentes grupos sociales no asentados en la actividad laboral, resultante del debilitamiento del valor del trabajo, así como insuficiencias en la socialización juvenil con impactos en otras áreas de los valores (Domínguez, 1996: 34).

Hay que partir por considerar que la magnitud de la caída económica que se produjo durante esos años implicó una drástica reducción de los niveles de vida de la población cubana, lo que ha significado una considerable afectación para los distintos

3 Esos rasgos fueron identificados en diferentes investigaciones realizadas en momentos previos y durante los años más intensos de la crisis económica, tales como "Las Generaciones en la Sociedad Cubana Actual" (Domínguez, Ferrer y Valdés, 1990); "Efectos del Período Especial sobre la Juventud" (Domínguez y Ferrer, 1993); "Las Identidades. Una Mirada desde la Psicología" (De la Torre, 2001), entre otras. 
grupos. Digamos, por ejemplo, que, en solo tres años (entre 1989 y 1992), el consumo per-cápita de los hogares se redujo en 18,5\% (ONE, 1997).

Pero también es necesario reconocer que una de las dimensiones principales de la estrategia de reajuste seguida se encaminó a repartir la crisis con equidad, es decir, evitar la toma de medidas puramente económicas que tuvieran un fuerte costo para algunos grupos en particular, como podría haber sido la racionalización laboral indiscriminada o mercantilizar los servicios sociales básicos, a la vez que se hicieron esfuerzos por compensar aquellos sectores más afectados a través de un reforzamiento de la seguridad social.

Por ejemplo, si se compara la proporción de ocupados dentro del total de personas en edad laboral en 1996 con la proporción de 1987, en los años más agudos de la crisis se produjo una reducción de apenas un $5 \%$ a pesar del crecimiento de ese segmento de la población en más de 650.000 personas (CEE, 1987; ONE, 1997). De igual forma, se reportó un crecimiento de los gastos en seguridad y asistencia social del 40\% entre 1990 y 1996, los cuales subieron del $17 \%$ al $24 \%$ del total de gastos del presupuesto (en la actividad no empresarial) (ONE, 1997).

Quiere decir que, aun en los peores momentos, se trató de conservar un nivel de justicia social que evitara el aplastamiento de ningún grupo.

Sin embargo, la naturaleza de la crisis y el tipo de salida que se fue configurando como posible en las circunstancias internas e internacionales en que esta ha tenido lugar, produjo inevitablemente un conjunto de efectos, algunos de carácter estructural, que tuvieron implicaciones para los niveles de integración social, en particular del grupo juvenil.

Elementos tales como la presencia de capital extranjero, el incremento de la actividad turística, el crecimiento de la activi- 
dad laboral por cuenta propia, la desestatalización de parte de la producción agropecuaria, la dualización de la moneda y la flexibilización de las regulaciones migratorias, fueron acciones, entre las más significativas, que dejaron sentir sus impactos. No es la intención hacer aquí el análisis de dichos cambios en sí mismos, sino hacer referencia a evaluaciones hechas desde las Ciencias Sociales de algunos de sus efectos sobre la juventud. En este trabajo, concentraremos la atención en dos cuestiones básicas: acceso a la educación y al empleo, como factores esenciales para el logro de la integración social.

Por ejemplo, la diversificación de las formas de propiedad con la apertura al capital extranjero, la creación de las UBPC (Unidades Básicas de Producción Cooperativa) y de otras formas de trabajo cooperativo, y el crecimiento del cuentapropismo, tuvieron importantes repercusiones sobre las condiciones de trabajo y de vida de sectores importantes y comenzaron a provocar un proceso de recomposición de la estructura de clases de la sociedad en el que, sin duda alguna, el componente generacional tuvo una particular relevancia. Ello dio lugar a distintos procesos con fuerte incidencia para la juventud tales como cambios en la estructura de ocupaciones en el sector formal de la economía, incremento de la subocupación, de los trabajadores por cuenta propia, de la desocupación y de la desvinculación del estudio y el trabajo, es decir, grupos de jóvenes que no se encontraban estudiando ni trabajando, pero que no podían considerarse desocupados porque no estaban buscando trabajo.

Aunque este fenómeno ya había tenido momentos altos en la segunda mitad de los años '80, al arribar a la edad laboral los nacidos durante la explosión demográfica de los años '60 (Domínguez, Ferrer y Valdés, 1990a), en el período de la crisis, las dificultades con la disponibilidad de empleos, sobre todo para los nuevos arribantes al mercado de trabajo, así como la insatisfacción con gran parte de las opciones existentes, el desequilibrio financiero que aquejó al país y la aparición de vías alternativas de 
obtención de ingresos no asociados al trabajo formal, incluido el nivel alcanzado por la economía sumergida, redujeron el interés de la juventud por alcanzar un empleo estable y elevaron la magnitud de los grupos de desvinculados.

Ya desde los primeros años (1991) se apreció la reducción de las tasas de actividad económica en las mujeres y la juventud (Ferriol, 1998). “... esta última condición [la inactividad] absorbió el $60,4 \%$ del crecimiento de los recursos laborales disponibles, por lo que solo poco más de un tercio de los arribantes fue a parar al mercado de trabajo" (Nicolau, 1999).

A su vez, según estudios realizados a mitad de la pasada década, el 79\% de una muestra representativa de jóvenes desvinculados laboralmente expresó tener quien los mantuviera y el 71\% no encontraba estímulo económico para trabajar (CESJ, 1995).

Esta situación comenzó a mostrar cierta reversión a partir de 1996, momento en que la desocupación alcanzó cifras entre 6\% - 7\% $\%^{4}$, de los cuales el $60 \%$ eran jóvenes, con mayor proporción de mujeres, calificación media o media superior y procedencia urbana (Valdés, 1997).

Estos procesos se dieron en estrecha relación con la esfera educativa, la que tuvo oscilaciones a lo largo del período y permitió el reforzamiento de algunas tendencias que ya se apuntaban desde etapas anteriores. Esta ha sido un área de análisis de gran relevancia y a la que la investigación social le ha dado un fuerte seguimiento por casi dos décadas de estudios, lo que ha permitido un monitoreo sistemático de la composición del estudiantado universitario y, por tanto, sus implicaciones para la estructura de clases de la sociedad (Domínguez, 2004).

Entre esas tendencias, se destaca la fuerte feminización de la enseñanza superior. A fines de los años '80, las muchachas cons-

4 Para esa fecha en América Latina en su conjunto se estimaba un 7,7\% de desempleo urbano, pero una elevada proporción de países superaba esa cifra (CEPAL, 1997). 
tituían el 43\% de estudiantes universitarios del país, pero, en la segunda mitad de los años ‘90 e inicios de la actual década, llegaron a representar más del 60\% del total (CEE, 1987; ONE, 2001). Ello evidenció los cambios producidos en los roles sociales de la mujer y los espacios de inserción social de que disponen. Pero, a su vez, alertó en el sentido de evitar situaciones que provocaran desventajas para algún grupo; en este caso, afectaciones para el ingreso en las universidades de los jóvenes varones.

Ello es solo una muestra de algunos efectos de interés para la juventud que se produjeron en el ámbito educacional, esfera altamente priorizada en las expectativas juveniles y que sufrió cierta desvalorización a lo largo de los años '90. No es posible dejar de considerar que la educación a lo largo de tres décadas sirvió como canal real de integración social a sectores socioclasistas y grupos raciales diversos y, en particular, actuó como factor dinamizador para la integración social de la mujer. Al finalizar la década de los años ‘ 80 , las niñas y mujeres constituían el 50,2\% de la matrícula total del país en los distintos niveles de enseñanza y el 57\% en el nivel superior (ONE, 1997).

La aspiración de estudios universitarios para la juventud se había hecho extensiva a toda la sociedad con independencia de la extracción social, el género, el color de la piel o la zona de residencia, pues se había afianzado, en la psicología social, como el mecanismo por excelencia de movilidad ascendente, tanto por su garantía para alcanzar un mayor nivel de vida como por constituirse en vía de realización personal y status social.

Si revisamos los resultados de la investigación realizada con una muestra nacional de personas pertenecientes a distintas generaciones y diferentes grupos sociales en la segunda mitad de los ‘ $80^{5}$, se aprecia que las aspiraciones de superación tenían un

5 Ese estudio sobre las generaciones en la sociedad cubana de ese momento, perteneciente al Programa Nacional sobre la Formación de la Juventud, abarcó a una muestra de 1687 jóvenes en seis provincias del país (Ciudad de la Habana, La Habana, Cienfuegos, Camagüey, Santiago de Cuba y Guantánamo) (Domínguez, Ferrer y Valdés, 1990). 
peso considerable que las hacía situarse en el primer lugar para el universo juvenil como conjunto $y$, aunque las diferencias entre los cinco grupos estudiados (clase obrera, trabajadores intelectuales, campesinos, estudiantes y desvinculados del estudio y el trabajo) fueron muy significativas, revela que, aun en aquellos grupos que ya se habían alejado de la actividad de estudio (ya fuera por haber concluido el nivel superior, como es el caso de los profesionales, o por que la actividad laboral no les exigía un mayor nivel de preparación, como es el caso de los campesinos) cerca de la décima parte mantenía ese tipo de deseos.

Otros indicadores también aportaron elementos acerca del peso del estudio en la vida de la juventud. En este caso, como origen de sus principales satisfacciones o preocupaciones, a la vez que la centralidad de la educación quedaba reforzada por la visión de la no-existencia de dificultades considerables para acceder a esta esfera (Domínguez, Ferrer y Valdés, 1990).

De manera que, aun cuando se hizo un gran esfuerzo por minimizar los impactos de la crisis y el reajuste sobre el área educacional y se logró conservar la cobertura educativa en la enseñanza primaria y secundaria a pesar de la menor disponibilidad de recursos, se produjeron efectos objetivos o estructurales, $\mathrm{cu}-$ yas tendencias más marcadas fueron las modificaciones de la estructura interna de la enseñanza media superior con la reducción de la enseñanza preuniversitaria, la ampliación de la enseñanza politécnica, sobre todo la agropecuaria y la potenciación de los preuniversitarios vocacionales como vías de acceso a las universidades. En el mismo sentido, se redujo la matrícula general en la enseñanza superior y, en particular, en algunas especialidades. Todos estos procesos contribuyeron a modificar la composición del estudiantado universitario, concentrándolo en hijos de profesionales, del sexo femenino y con escasa representación de jóvenes negros y mestizos.

Ello, a su vez, estuvo acompañado de efectos subjetivos en la percepción social del papel de la educación, una vez que esta 
dejó de ser el canal de movilidad social por excelencia al perder su espacio como principal vía de acceso a un mayor nivel de vida y, por tanto, esencial mecanismo favorecedor de status social, frente al surgimiento de otras vías de acceso a mayores niveles de ingreso (empleo en el sector emergente, trabajo por cuenta propia, remesas del extranjero, actividades ilícitas, etc.), aun cuando ese proceso no se dio de manera uniforme sino con variaciones a lo largo de la década y con notables diferencias entre grupos sociales.

El incremento de la heterogeneidad estructural y de la diferenciación que de ella se deriva ha producido una diferenciación en el área subjetiva, en particular, en cuanto a percepciones sociales, expectativas y valores, aunque pudo constatarse que la educación y el trabajo se encontraban entre las cuatro esferas básicas en las que se concentraban prioritariamente las aspiraciones, satisfacciones y preocupaciones de la juventud (familia, superación, trabajo y condiciones materiales de vida) (Domínguez, Cristóbal y Domínguez, 2002) ${ }^{6}$.

Se apreció también el predominio de una visión optimista acerca del logro de la mayor parte de las aspiraciones, lo que se atribuía principalmente al esfuerzo propio y la voluntad personal, conjuntamente con la existencia de condiciones sociales que lo favorecían. Sin embargo, aunque alcanzó mayor peso esta visión optimista, no puede obviarse el hecho de que, para una proporción de la juventud, se expresó cierto escepticismo en lo referente a la satisfacción de determinados tipos de aspiraciones sobre todo referidas al mejoramiento de condiciones materiales de vida individual.

6 La investigación a la que se hace referencia es un estudio cualitativo que abarcó una muestra de 436 jóvenes distribuidos en la región occidental y oriental del país en las provincias Ciudad de la Habana, Pinar del Río y Granma, pertenecientes a diferentes grupos sociales. Desde el punto de vista socio-ocupacional, se incluyeron obreros, profesionales y dirigentes de los distintos espacios (estatal tradicional, estatal reanimado y mixto); campesinos (privados y cooperativistas); trabajadores por cuenta propia; estudiantes de distintos niveles de enseñanza (preuniversitaria, politécnica y universitaria) y desvinculados del estudio y el trabajo. Se tomó en cuenta la distribución por sexos y por color de la piel. 
Fue significativo el alto valor que se le atribuyó a la capacidad de la juventud cubana para enfrentar problemas y resolverlos, trabajar y esforzarse, como principal rasgo de su identidad como grupo generacional. Aquí se enumeraron cuestiones tales como el interés por aprender, por estudiar, por lograr un objetivo, por prosperar, la capacidad para enfrentar problemas, el tener muchas ideas, ser ágiles, activos, dinámicos, preparados, perseverantes, trabajadores, luchadores, abnegados, emprendedores, firmes, cumplidores, responsables, creativos y con voluntad.

El estudio constató cierta heterogeneidad de percepciones de la juventud en relación con el mundo adulto y también en relación con los distintos segmentos etáreos que conforman la propia juventud. Conviven percepciones de predominio de diferencias y de semejanzas generacionales aunque las primeras superaron un tanto a las segundas. Sin embargo, la percepción de diferencias tampoco fue homogénea, pues se destacaron tres posiciones clave: una valoración comparativa que consideró que esa juventud tenía rasgos más positivos que los adultos; una segunda que, en la misma comparación, atribuyó las características más positivas a los adultos y una tercera que, sin hacer una evaluación ni colocar un signo, reconoció diferencias entre ambos, condicionados por los contextos.

De particular relevancia para la subjetividad juvenil resultó el hecho de que la juventud apreció, al interior de ella misma, mayores diferencias que similitudes, asociadas a factores sociales, culturales, valóricos y comportamentales, económicos y políticos, a pesar de que un segmento considerable apuntó la presencia de rasgos comunes asociados a las características de la edad que propicia gustos, intereses, aspiraciones y experiencias afines.

Ello explica por qué no se encontró una mayor identificación entre los miembros de los distintos subgrupos etáreos considerados jóvenes, especialmente la distancia entre el segmento de 25 a 30 años y el resto, que cuestiona la validez -ya puesta en duda desde finales de la década de los años ' $80^{7}$ (Domínguez, 1991)- de 
considerar al grupo juvenil dentro de límites tan extensos, diferencias que se han potenciado fuertemente si se tiene en cuenta el distinto significado de vivir los años '90 -como etapa de crisis económica y reajuste- en la infancia o en las distintas etapas de la propia juventud.

Asimismo, la lectura general de la subjetividad juvenil identificó cierta diferenciación según la pertenencia a distintos grupos sociales. Sin lugar a dudas, el grupo socioclasista y el territorio fueron, en ese orden, los dos factores decisivos de distinción, aunque el sexo y el grupo de edad (sobre todo entre el mayor y el resto, como ya se apuntó) marcaron importantes diferencias.

A pesar del nivel de heterogeneidad que mostraron los resultados de la investigación, fue posible encontrar puntos de engarce que hacen que la subjetividad de la mayor parte de los grupos que componen la juventud -con sus matices y diferencias internas- no resultara contradictoria con las principales metas de integración social de la sociedad cubana, aunque esa relación no se da de una manera lineal.

\section{La integración social de la juventud cubana en la primera década del siglo XXI. La atención de la política social}

La constatación y concientización de los cambios que se han operado en la estructura social cubana, particularmente en la estructura sociodemográfica, acompañados del crecimiento de las desigualdades sociales como resultado de los efectos que generaron más de una década de crisis económica y de reajustes en el modelo socioeconómico, indican nuevos retos a la política social.

7 En Cuba se consideran jóvenes las personas entre 14 y 30 años y a ellas van dirigidas las políticas sociales hacia la juventud. Sin embargo, desde fines de los años 80 , las investigaciones han constatado importantes diferencias en el subgrupo de mayor edad (25-30 años) que lo acercan más al grupo adulto, lo que sugiere una concentración de los procesos típicamente juveniles en el segmento que reconoce la ONU como juventud, es decir 14-25 años. 
Ello se inscribe en los propósitos planteados a partir del año 2000 de potenciar el desarrollo humano. Para esto, se han definido nuevas metas sociales a pesar de las dificultades económicas, lo que ha implicado la formulación de objetivos estratégicos y políticas sociales concretas y medibles, encaminadas a elevar la calidad de vida de la población y abrir nuevos espacios a grupos sociales específicos entre los que se encuentra con máxima prioridad la juventud. Para ello, se hace necesario "elevar la calidad de los servicios, brindar servicios más personalizados e incrementar su eficiencia" (INIE, 2005: 9).

Las principales prioridades quedan recogidas en un conjunto de Programas, de los cuales existen en la actualidad más de cien que forman parte de lo que se ha dado en llamar la "Batalla de Ideas" por la formación de una cultura general integral en la población y un mejoramiento de la calidad de vida.

En el caso de los programas sociales dirigidos a la juventud, se tomó en cuenta la presencia considerable de segmentos de jóvenes desocupados y desvinculados de las actividades educativas y laborales, de manera que se han creado diferentes opciones que permitan su incorporación a diferentes posibilidades de estudio y trabajo.

Dichos programas tienen, entre sus objetivos, recuperar el valor de la educación, brindar opciones para la continuidad de estudios que permitan una formación no solo técnica sino cultural integral y reinsertarlos en labores socialmente útiles. Permiten una formación emergente a través de cursos de formación profesional intensivos que los preparan para su incorporación al trabajo y para la continuidad de estudios superiores. Estos programas, a pesar de su corto tiempo de existencia, han comenzado a revertir algunas de las tendencias que predominaron en el decenio anterior y han reducido significativamente la desocupación y desvinculación juvenil.

Solo en el año 2002 se crearon 158.000 nuevos empleos prin- 
cipalmente en actividades vinculadas a la agricultura urbana y servicios básicos a la población como maestros primarios, profesores de computación, trabajadores sociales, enfermeros y operadores de salas de video. La tasa de desocupación para esa fecha se había reducido a la mitad con una cifra de 3,3\% (CEPAL, 2003). A inicios del año 2006, la cifra de desocupados se había reducido a menos del $2 \%$, lo que coloca a Cuba en condiciones de pleno empleo (Castro, 2006: 7).

En este propósito, ha incidido la consideración del estudio como empleo. Para ello, en el año 2001, se crearon los cursos de superación integral para jóvenes entre 18 y 29 años desvinculados del estudio y el trabajo, con remuneración y con posibilidades de continuar estudios en la educación superior. En los dos primeros cursos, ya habían egresado más de cien mil jóvenes y, de ellos, la tercera parte había ingresado en la educación superior (Castro, 2003).

Este plan se ha acompañado con la ampliación de la enseñanza superior a todas las localidades del país, a partir de la creación de Sedes Universitarias Municipales que facilitan la concurrencia a las aulas con planes de estudio especialmente habilitados para una atención directa al estudiante. Este programa ha permitido que, en solo cinco años, la matrícula de nivel superior haya crecido más de dos veces y media para alcanzar en el curso 2005-2006 un total de 510.000 estudiantes universitarios (Castro, 2006: 7), la mayor cifra alcanzada en la historia del país y que todos los graduados de bachilleres puedan acceder al nivel terciario de educación.

Aunque no es posible con los datos disponibles calcular con precisión la tasa de matrícula universitaria que esta cifra representa porque las edades de estos estudiantes no necesariamente se corresponden con las edades en que habitualmente se realizan estos estudios, se estima que debe oscilar entre el $25 \%$ - 30\%, lo cual coloca a Cuba entre los países de más alta tasa de América Latina y cerca del modelo de acceso universal al nivel terciario.

La creación de las sedes universitarias municipales, además 
de permitir ampliar la matrícula, han contribuido a modificar la composición social del estudiantado universitario, lo que amplía las oportunidades educativas a sectores más amplios de la sociedad, en particular jóvenes procedentes de grupos sociales con menores ventajas y evita el peligro de elitización de los profesionales. Por ejemplo, en el curso 2004-2005, mientras que en los cursos regulares diurnos el 79\% eran hijos de profesionales y el 63\% blancos, en las sedes municipales, el 77\% eran hijos de obreros y el 51\% negros y mestizos (Gómez, 2004).

Estos programas de Educación y Empleo para la etapa juvenil están acompañados de otros que crean las bases desde edades más tempranas, en niveles educativos previos, así como la potenciación de las acciones a través de mecanismos extraescolares. Por solo citar algunas de las acciones que están teniendo lugar, se pueden mencionar:

- Atención a los niños y adolescentes en educación primaria y secundaria a través de la reducción a 20 y 15 alumnos por aula respectivamente.

- Formación masiva de maestros emergentes de enseñanza primaria y profesores integrales de secundaria básica. Cambio en la concepción docente de la secundaria básica con el paso de profesores por asignaturas a profesores integrales.

- Reparación, ampliación y construcción de nuevas escuelas que mejore la infraestructura constructiva de la educación.

- Cambios en los programas de estudio con la introducción de la computación y programas audiovisuales en todos los niveles de enseñanza y garantía de su soporte tecnológico a través de la dotación a todas las escuelas de televisores, videos y computadoras.

- Creación de dos nuevos canales televisivos de corte educativo e introducción de programas como "Universidad para Todos" para la impartición de cursos especializados de diferen- 
tes materias, incluyendo idiomas extranjeros. Estos nuevos canales llegan ya a todas las provincias del país y abarcan al 87,9 \% de la población (Castro, 2006: 7).

- Ampliación del programa de los Joven Club de Computación y Electrónica a todas las localidades para contribuir a proporcionar una cultura informática a la comunidad con prioridad para niños y jóvenes. Existen ya alrededor de 600 instalaciones y se han graduado más de 800.000 personas (Granma, 2005).

\section{Consideraciones finales}

Los años '90, con su peculiar evolución para la sociedad cubana, han tenido impactos para la integración social de la actual generación joven. La misma se caracteriza por una mayor heterogeneidad estructural que las precedentes, a partir de cierta recomposición de la estructura socioclasista de la sociedad y del fortalecimiento de algunas diferencias territoriales asociadas al ritmo de recuperación económica y la presencia del sector emergente. De ello también se deriva el crecimiento de la heterogeneidad en el área subjetiva, en particular en cuanto a percepciones sociales, expectativas y valores, lo que se expresa en un amplio abanico de intereses y en una diversidad mayor que tiene sus efectos en la conformación de identidades, todo lo cual también impacta los procesos de integración social.

Los efectos diferenciadores de algunas de las medidas del reordenamiento económico, el incremento de la heterogeneidad de experiencias vitales acumuladas y la concentración de un segmento de la juventud en la búsqueda de salidas individuales que los aleja de la participación en soluciones colectivas, crean distancias al interior del grupo juvenil, limitando la conformación de esa identidad generacional ampliamente compartida.

Tampoco es posible desconocer las influencias más universales de la época, signada por la creciente interacción tecnológica y 
directamente humana, que imponen cambios y marcan la fisonomía de la actual generación joven con rasgos comunes más allá de fronteras nacionales. Estos procesos provocan efectos contrapuestos pues tienden, simultáneamente, a acentuar la fragmentación y a potenciar la integración al interior de la generación, lo que, a su vez, tiene impactos interesantes en la dinámica de las relaciones intergeneracionales.

De ahí que uno de los actuales retos de las políticas de juventud se oriente a la búsqueda de espacios reales de integración social acordes a las nuevas condiciones económicas y culturales -nacionales e internacionales- en las que se desenvuelven como generación y, para ello, la contribución de las investigaciones sociales resulta clave.

De los estudios realizados, se han ido derivando numerosas recomendaciones en la medida de su desarrollo, muchas de las cuales ya están siendo implementadas, principalmente en los Programas Sociales que se ejecutan actualmente. A ello se han añadido nuevas propuestas entre las que se encuentran:

- Utilización de los resultados sobre la subjetividad de la juventud para el diseño de su proceso de socialización en instituciones sociales tales como la escuela y los medios de comunicación.

- Consideración de las percepciones de la juventud en el diseño y evaluación de las políticas sociales a ella dirigidas.

- Seguimiento al desarrollo de estos procesos a partir del monitoreo sistemático a la evolución de un conjunto de indicadores sobre la integración social de la juventud.

A nuestro modo de ver, los objetivos clave que tienen las Ciencias Sociales Cubanas en la actualidad, en relación con el tema Juventud, son dos fundamentalmente:

- Caracterizar y evaluar qué efectos tienen, sobre los distintos 
grupos de la juventud, las transformaciones económicas y sociales que se llevan a cabo en el país, entendiendo su significado a la luz de la evolución histórica de dichos procesos.

- Proponer qué estrategias sociales deben desarrollarse para lograr la integración social de la juventud, que le permita actuar en las nuevas circunstancias con eficiencia social y satisfacción individual.

La complejidad de un empeño de tal naturaleza exige un esfuerzo colectivo a partir de una interpretación desde la óptica de diferentes disciplinas y la aplicación de un enfoque diferenciado para aquellos grupos que constituyen o constituirán actores sociales centrales en dichos procesos. Ello exige superar la actual dispersión de nuestros estudios y lograr una interconexión más efectiva.

\section{Bibliografía consultada}

Alarcón, Rodolfo (2004). Intervención del Vice - Ministro del Ministerio de Educación Superior en el taller "Universalización de la universidad". La Habana: Periódico Granma, 27 de marzo.

Castro, Fidel (2003). Discurso pronunciado en el acto de inauguración del curso escolar 2003-2004. La Habana: Periódico Granma, 9 de septiembre.

(2006). Discurso pronunciado con motivo del $1^{0}$ de Mayo. Suplemento Especial del Periódico Granma, La Habana.

CEE Comité Estatal de Estadísticas (1987). Anuario estadístico de Cuba. La Habana.

CEPAL (1997). Panorama social de América Latina 1996, Santiago de Chile. PanoramasocialdeAméricaLatina.1999-2000.http://www.eclac.cl (2003). Cuba: Evolución económica durante 2002 y perspectivas para 2003. (LC/MEX/L.566), 24 de julio.

CESJ Centro de Estudios sobre la Juventud (1995). "Estudio sobre los desvinculados laborales". Informe de investigación. La Habana.

Domínguez, María Isabel (1991). "Propuesta de reajustes de los rangos de edades de las organizaciones políticas y estudiantiles". Informe de investigación. La Habana: Centro de Investigaciones Psicológicas y Sociológicas (CIPS). 
(1995). "Las investigaciones sobre juventud en Cuba". Revista Temas, Vol. 1, La Habana.

(1996). "La formación de valores en la Cuba de los años 90: Un enfoque social", La formación de valores en las nuevas generaciones (Comp.). La Habana: Edit. Ciencias Sociales.

(2004). "Higher Education in Cuba: Democratization and the Role of Women". The Challenges of Public Higher Education in the Hispanic Caribbean. M.J. Canino and S. Torres - Saillant (Edit.). Markus Wiener Publishers, Princeton, 103 - 122.

(2005). "Cuban Youth: Aspirations, Social Perceptions and Identity". Changes in Cuban Society since the Nineties. Tulchin, J., Bobea, L., Espina, M y Hernández, R. (Edit.). Woodrow Wilson International Center for Scholars, Washington D.C., USA. 155- 167.

y María Elena Ferrer (1993). “Efectos del período especial sobre la juventud". Informe de investigación. CIPS. La Habana.

(1996). Jóvenes cubanos: Expectativas en los 90. La Habana. Edit. Ciencias Sociales.

María Elena Ferrer y María Victoria Valdés (1990). "Las generaciones en la sociedad cubana actual". Informe de investigación. CIPS. La Habana.

$\left(1990^{\mathrm{a}}\right)$. "Características generacionales de los estudiantes y los desvinculados del estudio y el trabajo". Informe de investigación. CIPS. La Habana.

Desirée Cristóbal y Deysi Domínguez (2000). "La integración y desintegración social de la juventud cubana a finales de siglo. Procesos objetivos y subjetividad juvenil". Informe de investigación. CIPS. La Habana.

(2002). "Subjetividad e integración social de la juventud cubana". Informe de investigación. CIPS. La Habana.

(2003). “Tendencias de integración social de la juventud en ciudad de la Habana". Informe de investigación. La Habana.

(2004). "Subjetividad e identidad de la juventud en la capital". Informe de investigación. CIPS. La Habana.

Ferriol, Ángela (1998). El empleo en Cuba (1980-1996). La Habana: Edit. Ciencias Sociales.

Fuentes, Mara (2000). "Subjetividad y realidad social. Un modelo psicosocial para su estudio". Revista cubana de psicología 3, Vol. 17, La Habana: 281-287. 
Gómez Cabezas, Enrique (2004). "Trabajo social a escala local. Objetivos, vías y métodos". Ponencia presentada al primer encuentro Cuba-China de ciencias sociales. La Habana.

Granma (Periódico). "Más de 800.000 graduados en Joven Club de Computación". 1 de agosto de 2005, La Habana.

INIE Instituto Nacional de Investigaciones Económicas (2005). Millennium Development Goals. Cuba: First Report, La Habana.

Maluf, Norma Alejandra (Marcia) (2000). "Participación e Identidad en el Consumo. Falsos Dilemas sobre lo Juvenil". Ponencia presentada en Reunión del Grupo de Trabajo sobre Juventud de CLACSO, San José de Costa Rica.

Martín Barbero, Jesús (2000). “Jóvenes: Comunicación e Identidad". Ponencia presentada a la Conferencia Iberoamericana de Ministros de Cultura. Ciudad de Panamá, septiembre 2000. Cumbres OEI - Cultura - Conferencia 2000. http:/ / www.oei.es

Medina Carrasco, Gabriel (2000). "La Vida se Vive en Todos Lados. La Apropiación Juvenil de los Espacios Institucionales". Aproximación a la Diversidad Juvenil. Gabriel Medina Carrasco (Comp.). México, El Colegio de México: 79-115.

Nicolau, José Luis (1999). “La Problemática del Empleo. Del Diagnóstico a los Servicios de Empleo". Informe de Investigación. CIPS. La Habana.

ONE Oficina Nacional de Estadísticas (1997). Anuario Estadístico de Cuba 1996. La Habana.

(2001). Anuario Estadístico de Cuba 2000, La Habana.

(2005). Censo de Población y Viviendas. Cuba 2002. Informe Nacional, La Habana.

Soares, Camilo (2000). "Aspects of Youth, Transition and the End of Certainties". International Social Science Journal 164. París: UNESCO, 209-218.

Torre, Carolina de la (1995). "Conciencia de Mismidad: Identidad y Cultura Cubana". La Habana: Revista Temas Vol. 2, 111-115.

(2001). Las Identidades. Una Mirada desde la Psicología. La Habana: Centro de Investigación y Desarrollo de la Cultura Cubana "Juan Marinello".

Valdés, Salvador (1997). "Economía y Empleo deben Marchar Juntos" (Entrevista al Ministro del Trabajo). La Habana: Periódico Granma, 3 de enero. 